\title{
Impact of Antenna and Propagation on Wireless Communication Technology: Terminal, Base Station, and Channel Modeling
}

\author{
Guangyi Liu, ${ }^{1}$ Cheng-Xiang Wang, ${ }^{2}$ Min Zhang, ${ }^{3}$ James Warden, ${ }^{4}$ and Huanhuan $\mathrm{Gu}^{5}$ \\ ${ }^{1}$ Wireless Department of China Mobile Research Institute, Beijing, China \\ ${ }^{2}$ Joint Research Institute for Signal and Image Processing, School of Engineering \& Physical Sciences, Heriot-Watt University, \\ Edinburgh, UK \\ ${ }^{3}$ Alcatel-Lucent Telecom Ltd., The Quadrant Stonehill Green, Westlea, UK \\ ${ }^{4}$ Research In Motion Ltd., USA \\ ${ }^{5}$ Research In Motion Ltd., Canada
}

Correspondence should be addressed to Guangyi Liu; liuguangyi@chinamobile.com

Received 14 March 2013; Accepted 14 March 2013

Copyright (C) 2013 Guangyi Liu et al. This is an open access article distributed under the Creative Commons Attribution License, which permits unrestricted use, distribution, and reproduction in any medium, provided the original work is properly cited.

For wireless communication, the antennas at both sides of the wireless chain and the corresponding propagation characteristics play an important role in the system design and optimization. In past standardization activities, for example, in 3GPP LTE/LTE-Advanced and IEEE $802.16 \mathrm{~m}$, only some simplified antenna patterns are assumed during the standardization, which may influence the system parameter optimization and system performance for practical deployment. For example, only omnidirectional antenna pattern is assumed at the terminal, and perfect sectoring antenna pattern is assumed at the base station, while the impact of practical antenna pattern, coupling among the antennas, and human body on the channel characteristics have been ignored. It is therefore valuable to evaluate how these nonideal issues on the antennas will influence the propagation characteristics and thus the system design. This special issue has accepted 8 papers as below.

The first paper investigates the positive effect of severe Nakagami- $m$ fading on the performance of multiuser Transmit Antenna Selection/Maximal-Ratio Combining (TAS/ $\mathrm{MRC}$ ) systems with high selection gain. Both amount of fading (AF) and symbol error rate (SER) of M-QAM are derived as closed-form expressions for integer $m$. For arbitrary $m$, the AF and the SER are expressible as a single infinite series of Gamma function and Gauss hypergeometric function, respectively. The analytical results lead to the following observations. First, the SER performance can demonstrate the positive effect of severe Nakagami-m fading on multiuser TAS/ MRC systems with high selection gain. Second, the AF performance only exhibits the negative impact of severe fading regardless of high selection gain. Last, the benefit of severe fading to the system performance diminishes at high signalto-noise ratio (SNR).

In the second paper, an array of monopoles antenna over a ground plane that radiates a directive lobe in the endfire direction is described. The design uses the rigorous method described in order to synthesis the radiation though the strong cumulative coupling between the monopoles. A gain higher than $20 \mathrm{~dB}$ was achieved in the end-fire direction over a $4.5 \%$ bandwidth. However, the antenna has been tilted in order to compensate the beam deviation caused by the edge diffraction. A prototype with 12 elements has been manufactured in order to validate the antenna principle and the whole antenna is successfully measured.

In the third paper, user influence on multiple-input multiple-output (MIMO) performance is studied for different dual antenna handsets specially designed to have good and bad MIMO performance. The study reveals that user influence can cause either improvement or degradation for different test objects, including a spread effect over the parameters. 
Differences in performance between good and bad handsets can be clear when they are measured without user influence, but become small under real person influence. This result illustrates the particular importance of user influence to characterize MIMO handsets.

The fourth paper focuses on the fading characteristics of wireless channel on high-speed railway (HSR) in hilly terrain scenario. From the measurement conducted on the Guangzhou-Shenzhen passenger dedicated line in China with the speed of $295 \mathrm{~km} / \mathrm{h}$ at $2.4 \mathrm{GHz}$, the fading parameters, including path loss, shadow fading, and $K$-factor, are analyzed. Compared with the free space path loss model, the measured path loss value in hilly terrain scenario suffers greater decay and shows the property of having a breakpoint distance as the WINNER HSR model shows. The shadow fading is lognormal fitting and changed with two turning points. the mean value of decorrelation distance is around $14 \mathrm{~m}$. But the value will rise up when getting near to the huge reflectors. The $K$-factor as a fast fading parameter is also influenced by the breakpoint distance. Within the breakpoint distance, $K$-factor decreases as the $T$ - $R$ distance increases. Beyond the break point distance, the value of $K$ factor fluctuates dramatically.

In the fifth paper, to evaluate the sector antenna performance degradation, three performance indicators are defined as the normalized "In Sector Power Gain" (ISPG), the normalized "In Sector Mean Power Gain" (ISMPG), and the "In Sector Standard Deviation of the Power Gain" (ISSDPG). Then the antenna performance degradation in three conventional wireless access point scenarios of small cell (antenna wall, antenna shelter, and antenna-lamp post) is investigated for directional or omnidirectional antennas. The results indicate that the lognormal distribution (Gaussian in $\mathrm{dB}$ ) is often approximately obeyed, as regards the power gains in the considered sector. This is true for the antennawall scenario, while in the antenna-shelter and antenna-lamp post scenarios, the ISPG appears to follow the generalized Gaussian distribution. The ISSDPG follows well a lognormal distribution in the antenna-wall scenarios, within the frequency band from $1.7 \mathrm{GHz}$ to $2.0 \mathrm{GHz}$ for both directional and nondirectional antennas.

In the sixth paper, the impact of antenna separation and angle-of-arrival (AOA) spread on frequency domain adaptive antenna array (FDAAA) receiver and hybrid frequency reuse factor are analyzed. Large AOA spread can increase the cellular capacity, and increasing the antenna separation to above $5 \lambda$ can greatly increase the cellular capacity. The optimal frequency reuse factor (FRF) depends on the channel propagation and transceiver scheme as well. Hybrid FRF algorithm can effectively improve the cellular outage capacity and therefore hybrid FRF together with FDAAA receiver is a good solution for uplink transmission in cellular system.

In the seventh paper, the modeling methodology that the antenna pattern and antenna constellation could be incorporated into propagation channel model independently by receiver antenna and transmitter antenna is validated by the field channel measurement in indoor scenarios at $2.35 \mathrm{GHz}$. First, the $2 \times 2$ MIMO channel impulse responses (CIRs) is recorded with practical dipole antenna (DPA) and real terminal antenna as the reference. Second, the CIRs are reconstructed from the available International Mobile Telecommunications-Advanced (IMTAdvanced) channel model with the field patterns of test antennas and updated spatial parameters extracted from the similar measurement, in which the omnidirectional antenna array (ODA) is used at both sides of the transmission link to capture the omnidirectional spatial information. The comparisons between field data and reconstructed data are made from coherent bandwidth, eigenvalue dispersion, outage capacity, and ergodic channel capacity. It is found that the reconstructed data closely approximates real data in coherent bandwidth and correctly describe the statistical characteristics in frequency domain. But difference is found in the spatial correlation, which causes the underestimation of the $5 \%$ channel outage capacity. So the modeling method which ignores the nonideal issues on the antennas will have impact on the characteristics of MIMO channel, especially on the spatial characteristics.

In the eighth paper, the influence of correlated noises on the Maximal-Ratio Combining (MRC) diversity gain and multiple-input multiple-output (MIMO) capacity is investigated. The noise correlation effects on diversity gains and capacities are formulated using the noise prewhitening concept, and the computational robustness of the derived MRC CDF was proved rigorously. The effects of correlated noises on MRC diversity gains and MIMO ergodic capacities were studied via reverberation chamber measurements. It was shown that, only for multiport antennas with very small correlations, the noise correlation effects on diversity gains (capacities) can be neglected. Otherwise, it should be taken into account for accurate diversity (capacity) measurements.

Guangyi Liu Cheng-Xiang Wang Min Zhang James Warden Huanhuan Gu 

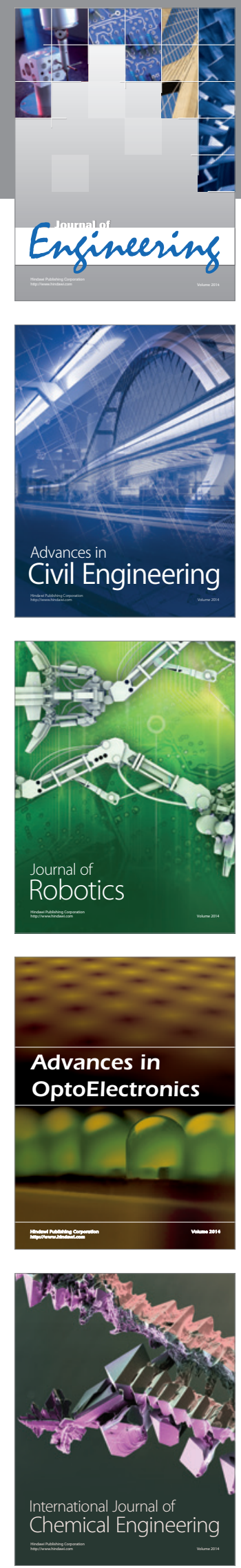

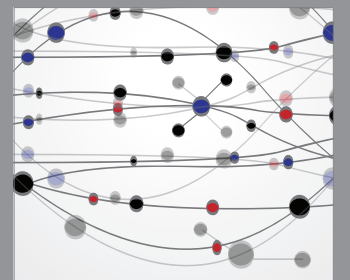

The Scientific World Journal
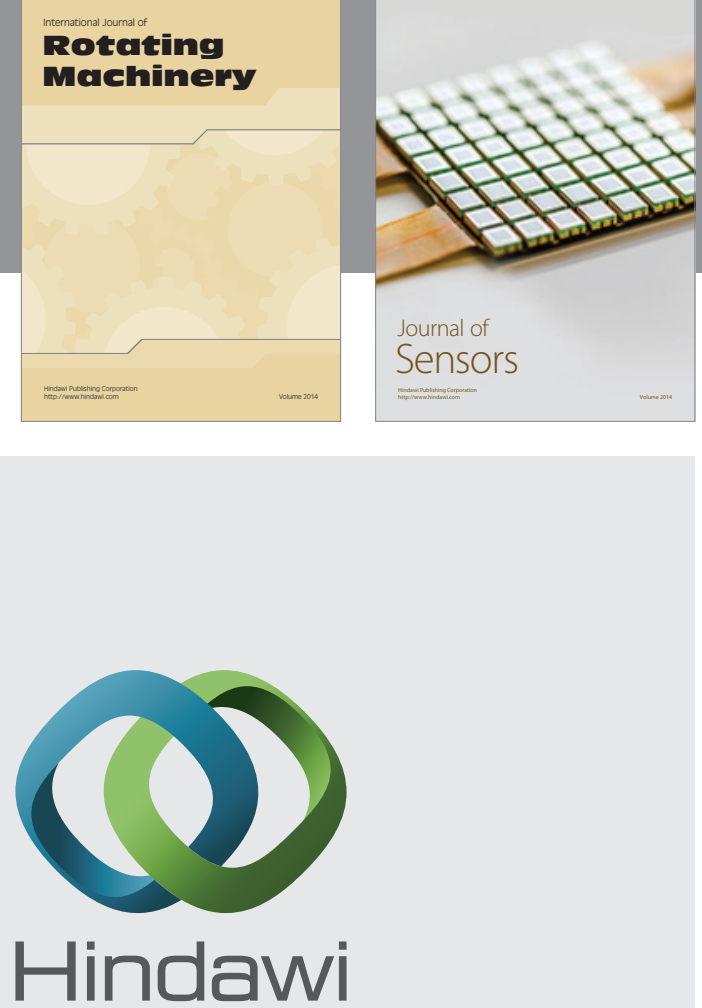

Submit your manuscripts at http://www.hindawi.com
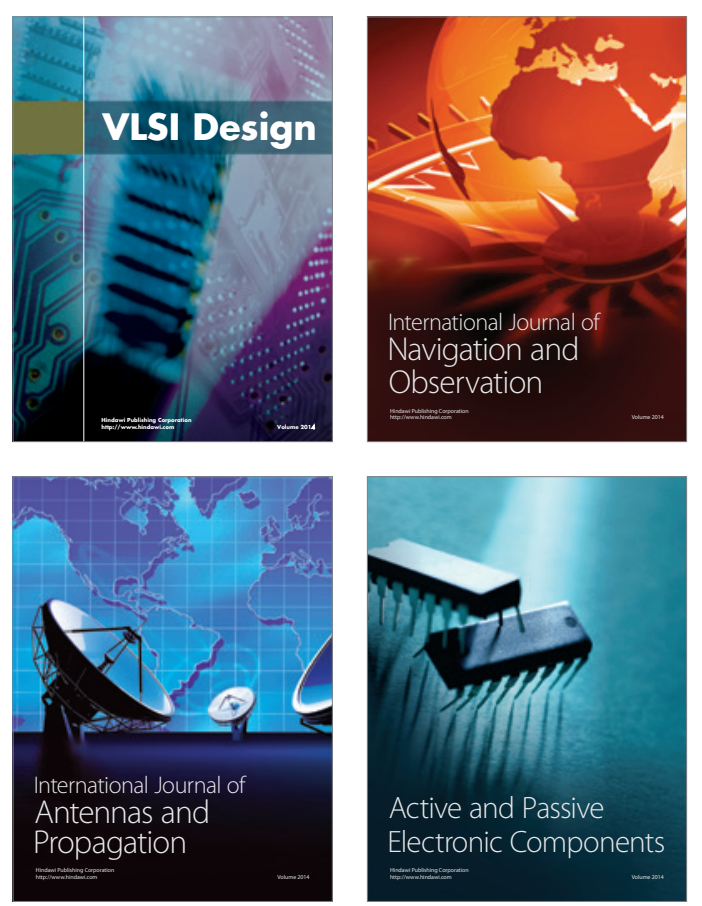
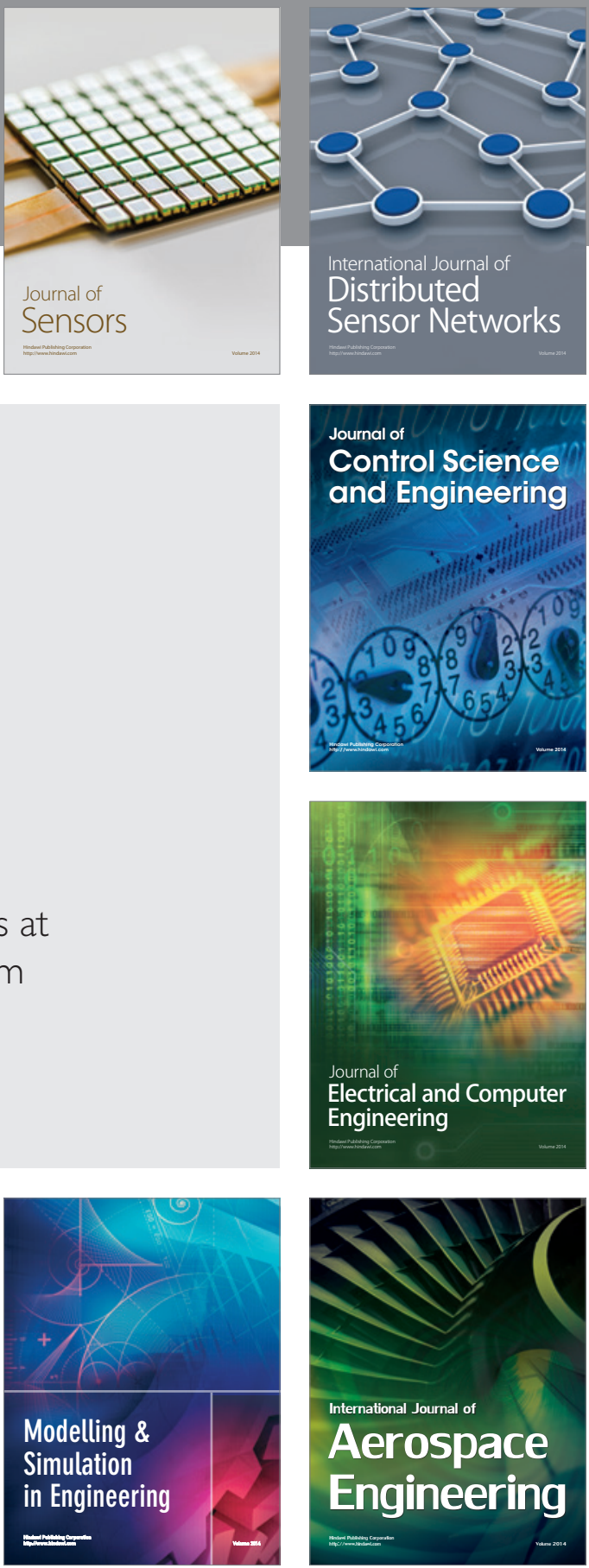

Journal of

Control Science

and Engineering
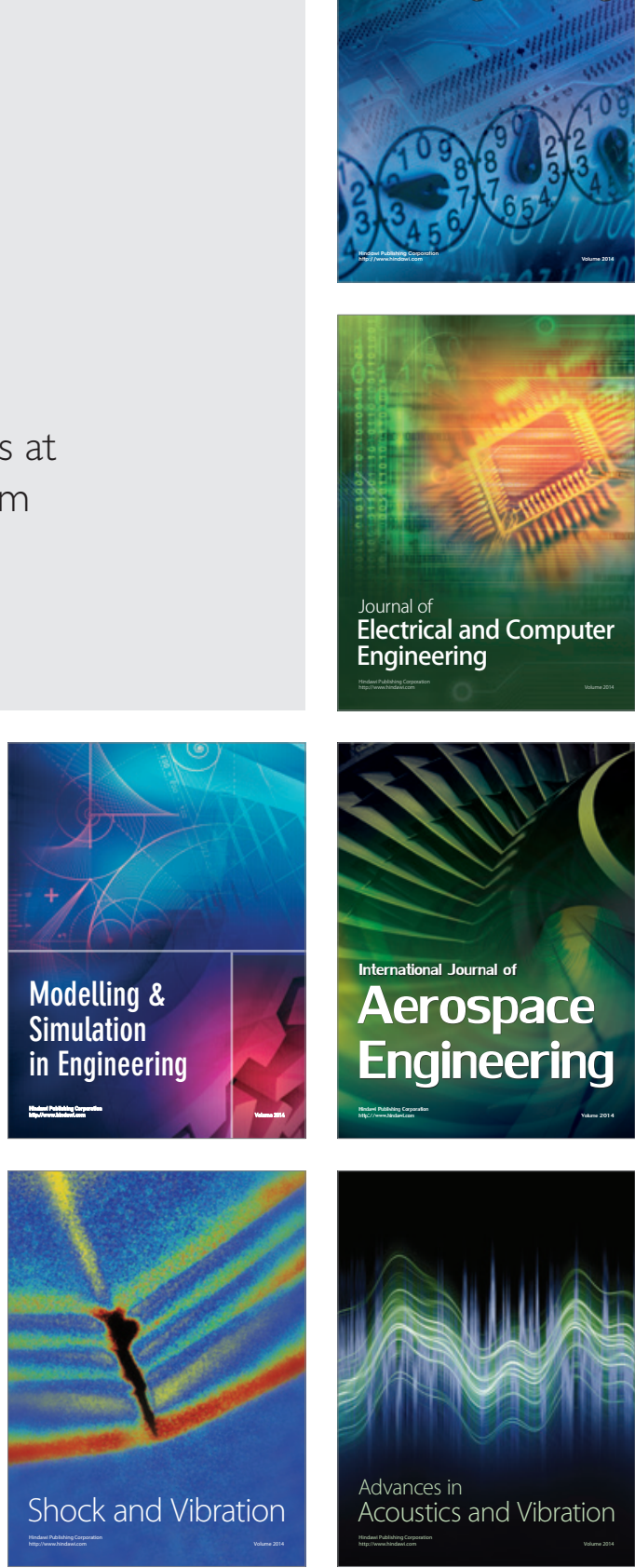